\title{
Wie fremd müssen Helden sein? Überlegungen zu Othello
}

\author{
Tobias Döring
}

1.

Vor fünfzig Jahren erschien bei Oxford University Press ein Buch mit dem Titel Othello's Countrymen, eine auch in neuerer Forschungsliteratur noch gelegentlich zitierte Pionierstudie, ${ }^{1}$ die sich mit The African in English Renaissance Drama, so der Untertitel, auseinandersetzt. Ihr Pionierstatus gründet nicht so sehr in ihrem Gegenstand; zu diesem Thema war auch zuvor schon einiges erschienen. Vielmehr gründet er in der Person des Autors, Eldred Durosimi Jones, prominenter Literaturwissenschaftler des postkolonialen Afrika, mit dem hier erstmals ein Afrikaner zur Figur der Afrikaner im englischen Renaissancetheater, für die Othello prototypisch einsteht, Stellung nahm. Jones, Jahrgang 1925, war aus Sierra Leone, lehrte dort als Professor am Foray Bay College der University of Sierra Leone, war einige Jahre auch deren Rektor und lange einer der führenden Köpfe der literarischen wie kulturpolitischen Szene jener großen Aufbruchszeit. ${ }^{2}$ Das mag seiner Sicht auf Shakespeares Bühnenafrikaner in den Augen mancher Leser besondere Bedeutung, vielleicht sogar authentische Kennerschaft verliehen haben, obwohl sie gerade nicht auf Typisches hinausläuft: ,in the end“, erklärt Jones, „Othello emerges, not as another manifestation of a type, but as a distinct individual who typified by his fall [...] the weaknesses of human nature". ${ }^{3}$

Bei so viel Augenmerk aufs Individuelle wie auf das Menschlich-Allzumenschliche, das Othello offenbar verkörpert, wird allerdings die Frage interessant, auf welches Kollektiv der Titel von Jones' Studie letztlich zielt: Othello's Countrymen bezeichnet eine Landsmannschaft, deren Bestimmung alles andere als trivial ist. Zunächst meint er wohl den Gegenstand der Untersuchung, also die Gesamtheit der Schwarzen auf der Shakespeare-Bühne, darunter Aaron in Titus Andronicus, den Prinzen von Marokko in The Merchant of Venice und einige weitere, die alle offenbar als ,Landsleute' Othellos gelten sollen. Wenn diese aber nun erstmals von einem Afrikaner in einer gelehrten Abhandlung erforscht werden, weitet sich die Referenz des Titels und weist auch auf den Autor selbst, der ebenfalls zum so markierten

1 Siehe zum Beispiel Virginia Mason Vaughan: Othello. A Contextual History. Cambridge 1994, 60,64 .

2 Die Angaben folgen Werner Arens [et al.] (Hg.): Grundlagen zur Literatur in englischer Sprache, Bd. 5: Dieter Riemenschneider (Hg.): Grundlagen zur Literatur in englischer Sprache: West- und Ostafrika. München 1983, 114.

3 Eldred Durosimi Jones: Othello's Countrymen. The African in English Renaissance Drama. Oxford $1965,87$. 
Kollektiv gehören mag. Denn als Moor of Venice könnte Shakespeares Othello ein Modell bilden, um aktuelle Spannungen von Zugehörigkeiten auszuhandeln und zugleich Ansprüche auf Teilhabe an solcherlei Verhandlungen zu reklamieren. Als Othellos countrymen fordern Jones und Seinesgleichen mittels Shakespeares schwarzer Bühnenmaske vielleicht Anerkennung für die eigene Diskursposition; auch in der Shakespeare-Forschung und auch bei Oxford University Press ist künftig mit ihnen zu rechnen.

Das Beispiel jedenfalls macht Schule. Fünf Jahre nach Jones veröffentlicht John Pepper Clark, sein nigerianischer Kollege von der University of Lagos, Jahrgang 1935, bei Longman The Example of Shakespeare, eine Studie, in der Othello ebenfalls eine Schlüsselrolle spielt. Allerdings geht es hier nicht um das Renaissancetheater, sondern um aktuelle afrikanische Literatur und die Frage, wie diese sich artikulieren solle, das heißt, ob sie in der Sprache der ehemaligen Kolonisatoren wie Englisch oder besser in einer indigenen Sprache wie Yoruba abzufassen sei. Als Maßgabe für diese Grundsatzdebatte empfiehlt Clark, durchaus überraschend, Shakespeare nachzueifern, „the man everybody calls master", ${ }^{4}$ denn auch dieser habe seinen künstlerischen Sprachgebrauch an kulturelle Fremdheit angepasst: Wenn er außereuropäische Figuren auf die Bühne bringe, sprechen diese zwar durchgehend Englisch, allerdings in einer Weise, die ihre fremde Position berücksichtige und ihren kulturellen Eigenwert durch Bildersprache und Vergleiche deutlich mache. Diese Strategie sei zeitgenössischen postkolonialen Autoren Afrikas anzuraten: ins Medium des Englischen kulturelle Differenzen einzuschreiben. Vorbildlich dafür sei allerdings nicht Shakespeares Moor, sondern Shakespeares wilder Sklave aus The Tempest, also Caliban, weil dieser am nachhaltigsten durch verbale Eigenheiten kenntlich werde. Othello dagegen übernehme die Diktion der Herrschaft und sei kein brauchbares Modell, denn er repräsentiere eher „displaced persons, transplanted to a strange terrain in the manner of the American Negro". ${ }^{5}$ Seinen ,Landsleuten' in Afrika - um Jones' Formulierung aufzugreifen - gilt er daher als Kontrastbeispiel.

Diese Othello-Konzeption wird dominant. Als Inbegriff des Angepassten, Konvertierten, seiner Herkunft wie Kultur Entfremdeten figuriert Othello immer wieder in der Literatur jener Zeit - beispielsweise in Season of Migration to the North (1966), Tayeb Salihs sudanesischem Migrationsroman, der seit seiner englischen Übersetzung von 1969 zu einem postkolonialen Klassiker geworden ist, oder in Water With Berries (1971), George Lammings Diasporaroman über schwarze Künstler in England, der sich gleichermaßen kritisch wie emphatisch mit dem Shakespeare-Erbe auseinandersetzt, oder auch in Charles Marowitz' amerikanischer Bühnenadaption An Othello (1972), in der Iago als Black Power-Aktivist einem assimilierten „Hausneger ${ }^{6}$ schwarzes Bewusstsein einflößen will. ${ }^{6}$ Immer wieder

John Pepper Clark: The Example of Shakespeare. London 1970, x.

Ebd., 7.

6 Tayeb Salih: Season of Migration to the North, übers. von Denys Johnson-Davies. Harmondsworth 2003. George Lamming: Water with Berries. London 1971. Zur Bühnenadaptation 
dient Othello solcherart als Figur der Aus- und Abgrenzung zur Selbstpositionierung gegenüber den politischen Entscheidungen, für die sie steht oder jedenfalls in Anspruch genommen wird. Ein Held ist Othello diesen schwarzen Lesern deshalb gewiss nicht, auch kein Negativheld oder Schurke, eher eine Verlegenheit: Gerade wenn und weil sie sich mit Shakespeare als Inbegriff des Kolonialen und Kanonischen einlassen, mag ihnen wohl Shakespeares Bühnenfigur solcher Einlassungen und deren tragische Geschichte besonders beziehungsreich wie kritisch scheinen. In dem Maße zumindest, wie Othello einem country zugerechnet wird, gerät er seinen countrymen offenkundig zum Problem.

Das führt folgerichtig zur Frage, die dieser Beitrag an Othello stellen und erörtern will: Wie fremd müssen Helden sein? Wenn der Titel des vorliegenden Sammelbandes einlädt, über den Zusammenhang von Heldentum und Fremdheit nachzudenken, könnte damit, so die Leitthese, eine prinzipiell notwendige Verbindung angesprochen sein - und zwar nicht etwa, weil Helden uns, die wir zur „postheroischen“ Gesellschaft im Sinne Herfried Münklers zählen, ${ }^{7}$ ziemlich fremd geworden sind, sondern weil womöglich das Heroische konstitutiv an Alterität gebunden ist. Das jedenfalls scheint ein Kennzeichnen, vielleicht sogar ein Nenner, der diversen Überlegungen zur Konzeption von Heldentum zu sein, wie sie sich in den Grundsatzbetrachtungen des Freiburger Sonderforschungsbereichs finden: ${ }^{8}$ Stets geht es darum, Heldenstatus zwischen Exemplarischem und schlechthin Außerordentlichem zu begreifen, eine prekäre, wenn nicht paradoxe Konfiguration, in der dem Fremden eine Schlüsselfunktion zukommt.

Heldentum verlangt ein Narrativ, denn nur durch „literarische Verdoppelung“, so Münkler, ${ }^{9}$ lässt sich aus dem Gewalttäter ein Held machen. Dazu ist weiterhin ein Kollektiv erforderlich, das ihn durch Strategien des Imaginierens und Erinnerns formt und dabei auf spezifische Bedürfnislagen antwortet. Ohne diese funktionale Relation zwischen der herausgehobenen Figur und der Bezugsgruppe, die sich von ihr erzählt, fehlt Helden nicht nur Relevanz, sondern mutmaßlich jede Existenz. Doch in der narrativen Ausgestaltung der Beziehung zeigt sich der erwähnte Widerspruch: Einerseits will sich das Verehrerkollektiv in seinen Helden wiederfinden und -erkennen, andererseits verlangt es von ihnen Exzeptionalität. In Heldenerzählungen figuriert Vertrautes daher stets in unauflöslicher Verschränkung mit dem Fremden, da sie ein Dispositiv des Alltagstauglichen, Ordnungs-

durch Marowitz siehe Lois Potter: Othello (Shakespeare in Performance). Manchester 2002, 171.

7 Herfried Münkler: Heroische und Postheroische Gesellschaften. In: Merkur 61: 7, 2007, $742-752$.

8 Ralf von den Hoff [et al.]: Helden - Heroisierungen - Heroismen. Transformationen und Konjunkturen von der Antiken bis zur Moderne. Konzeptionelle Ausgangspunkte des Sonderforschungsbereichs 948. In: helden. heroes. héros. 1: 1, 2013, 7-14, DOI 10.6094/helden. heroes.heros/2013/01/03.

9 Münkler: Heroische und postheroische Gesellschaften (Anm. 7), 743. 
und Modellhaften mit einem Dispositiv des Außerordentlichen, Transgressiven kontern oder kreuzen müssen. Diese Spannung ist im Übrigen auch konstitutiv für Max Webers Konzept des Charismatikers, der von der Akklamation einer Gruppe in gleicher Weise abhängt wie die Gefolgsleute von seiner Führerschaft; ${ }^{10}$ dafür erscheint das Nichtvermittelbare, Nichtfassbare seiner Fremdheit geradezu als Bedingung und muss sich doch, um Bestand zu haben, einer „Veralltäglichung", so Weber, in herkömmliche Machtformen beugen.

Solchen Wechselverhältnissen in der Konfiguration des Heroischen will ich im Weiteren anhand jenes Tragödienhelden nachgehen, dessen irritierende Fremdheit sein Epitheton the Moor of Venice bereits vielsagend ausweist: ein Genitiv, der zwischen Exklusion und Exklusivität bis hin zu Zugehörigkeit und Projektion ein ganzes Spektrum möglicher Lesarten eröffnet. Wie also steht Othello in und zu Shakespeares Venedig und wie Venedig zu ihm? Was erzählt Venedig sich von ihm, und was erzählt er selbst? Welche Wirkung und Funktion haben solche unterschiedlichen Erzählungen im Theater? Und als was für einen Helden weisen sie Othello letztlich aus?

Shakespeare meidet das Wort hero im Gesamtwerk geradezu ${ }^{11}$ und zeigt doch immer wieder, wie es als Konzept mitspielt - nicht zuletzt, wenn er seinen Othello, wie Manfred Pfister schreibt, ${ }^{12}$ als strahlenden „Kriegsheld in venezianischen Diensten“ antreten, als „Liebesheld“ aber furchtbar scheitern lässt. Die folgenden Bemerkungen dazu richten sich auf wenige Aspekte im Dramentext und die komplexen Interaktionen, die er entwirft, bevor sie zum Schluss noch einmal kurz auf die Rezeptions- und Bühnengeschichte zurückkommen. Die Diskussion geht aus von dem Befund, dass dieses Stück sein Heldeninteresse bezeichnenderweise fast vollständig in narrative Vermittlung, kaum je in mimetische Darstellung umsetzt und zwar ausdrücklich auch, was Othellos eigentlich prägende Rolle als strahlender Kriegsheld angeht. Damit, so die zentrale Diskussionslinie, erweist Shakespeares Theater sich als dramatische Heldenverwertungsanstalt, um Strategien der Heroisierung und Deheroisierung ebenso durchzuspielen wie auch kritisch zu befragen.

10 Siehe dazu Eva Horn: Introduction. In: New German Critique 38: 3 (Themenheft: Narrating Charisma), 2011, 1-16.

11 So Manfred Pfister: Zur Einführung. Helden-Figurationen in der Renaissance. In: Achim Aurnhammer und Manfred Pfister (Hg.): Heroen und Heroisierungen in der Renaissance (Wolfenbütteler Abhandlungen zur Renaissanceforschung; 28). Wiesbaden 2013, 13-26, hier 23.

12 Ebd., 25. 
2.

Zum Ende mündet die Tragödie ins Erzählen; die letzten beiden Verse lauten:

Myself will straight aboard, and to the state

This heavy act with heavy heart relate. (V 2, 370-371) ${ }^{13}$

So der Senatsvertreter Lodovico, Repräsentant des venezianischen Gemeinwesens, der auf dem Außenposten Zypern die schrecklichen Ereignisse, die dort vorgefallen sind, besichtigt. Jetzt ist das Blut vergossen, die Leichenschau beendet und der Gefangene zur peinlichen Befragung abgeführt, da kündigt dieser Venezianer an, dass er sich einschiffen und zu Hause ,dem Staat ${ }^{`}$ Bericht erstatten will. Zum Ende also geht, was wir im Verlauf des Stücks gesehen haben, in eine narrative Ordnung über und kann auch erst in dieser Form, weil jegliches Geschehen transitorisch ist, ins kulturelle Gedächtnis eingehen. Das ist ein charakteristischer und oft erprobter Übergang (auch Hamlet endet ja mit einer solchen Geste: „All this can I| Truly deliver" erklärt Horatio [V 2, 369-370] ${ }^{14}$ ), wenn eine Handlung endet und eine Erzählung beginnt, wenn auf Aktion also Reflexions- und Relationsakte folgen. Es ist zugleich der Punkt, an dem alle Heldenkonstruktion ansetzt; „soziale Verbände sind nur dann heroisch“, lesen wir bei Münkler, „wenn sie über eine das Heroische reflektierende Literatur verfügen - zumindest wenn es umherziehende Sänger gibt, die mündlich tradieren, was die Helden geleistet haben". ${ }^{15}$ Diese Rolle nimmt zum Ende von Othello Lodovico wahr. Seine Ankündigung, die Segel zu setzen, um seine Botschaft zu überbringen, verweist auf den Beginn klassischer Ependichtung mit ihrer topischen Verbindung von Segeln und Singen; ${ }^{16}$ seine Formulierung „to the state | This heavy act [...] relate" bezeugt zudem, dass es darum geht, eine Beziehung zu stiften zwischen dem ,Akt und dem ,Staat', dessen Normen und Gesetze dem Vorgefallenen Bedeutung zuweisen; erst ein solcher Deutungsrahmen lässt Kontingentes zur Geschichte werden und sich potenziell um die Figuren als deren Schurken oder Helden ordnen.

Lodovico nimmt dabei zugleich die Worte und den letzten Willen Othellos auf, der in seiner Abschiedsrede ${ }^{17}$ genau diese Stichworte („state“, „deeds“, „relate“ [V 2, 338, 340]) gegeben, Lodovico also ausdrücklich zu seinem Berichterstatter ernannt und sehr genaue Anweisung erteilt hat, wie von ihm und seinen schrecklichen Verfehlungen zu sprechen sei, als wolle er sein postumes Gedenken auf die-

13 Alle Zitate aus dem Stück werden durch Angabe von Akt-, Szene- und Verszahl in Klammern nachgewiesen, gemäß folgender Ausgabe: William Shakespeare: Othello. The Oxford Shakespeare, hg. von Michael Neill. Oxford 2006.

14 William Shakespeare: Hamlet. The Arden Shakespeare, hg. von Ann Thompson und Neil Taylor. London 2006.

15 Münkler: Heroische und postheroische Gesellschaften (Anm. 7), 743.

16 Siehe Ernst Robert Curtius: Europäische Literatur und lateinisches Mittelalter. Tübingen und Basel ${ }^{11} 1993,138$.

17 Sie wird im vorliegenden Beitrag zum Ende des dritten Abschnitts vollständig zitiert. 
se Weise selbst bestimmen. Was hätte Lodovico denn auch seinerseits zu sagen? Was, abgesehen von dem „heavy heart“", das er hervorhebt, qualifiziert ihn eigentlich für diese Rolle als Vermächtnissprecher? Anders als Horatio, immerhin ein Intimus der Titelfigur und in vielen Szenen der Hamlet-Tragödie präsent, tritt Lodovico überhaupt erst im IV. Akt in Erscheinung und bleibt für die gesamte Handlung randständig. Was weiß er daher überhaupt von den Ereignissen auf Zypern und von den schrecklichen Verstrickungen, in die Othello dort geraten ist?

Eine solche Frage aufzuwerfen, heißt, nach dem Verhältnis des Titelhelden zu dem Kollektiv, das ihn bestimmt, zu fragen sowie grundsätzlich nach dem Konzept von Heldentum, das hier entwickelt wird. Zweimal zuvor ist Lodovico aufgetreten, zu Beginn des IV. und des V. Akts, und beide Male wird er Augenzeuge schier unglaublicher Gewaltausbrüche unter den Funktionseliten der venezianischen Armee, verstörende Beobachtungen, die seinen Auftrag als Senatsberichterstatter geradezu gefährden:

[...] this would not be believed in Venice,

Though I should swear I saw't. (IV 1, 233-234)

So stellt er fest, als er mit ansehen muss, wie Othello, immerhin Oberbefehlshaber der Streitkräfte, öffentlich seine Frau schlägt. Lodovico hat ihm eben den Brief des Senats überbracht, der ihn von diesem Amt entbindet, und muss jetzt feststellen, dass es dazu allerhöchste Zeit ist:

Is this the noble Moor, whom our full Senate

Call all in all sufficient? (IV 1, 256-257)

Das Gemeinwesen erkennt nicht mehr, wem es sein Schicksal in die Hände legte, und sein Sprecher weiß zum Schluss nicht mehr, wie er von diesem destruierten, deheroisierten Helden sprechen soll:

O thou, Othello, that was once so good, [...]

What shall be said to thee? (V 2, 289-291)

Othellos Antwort ist bezeichnend für das in Rede stehende Problem:

Why anything -

An honourable murderer, if you will (V 2, 291-292)

Die Rede vom ,ehrenwerten Mörder', die hier als Nachrufformel angeboten wird, folgt der rhetorischen Verlegenheit, dass offenkundig Widersprüchliches zusammengebracht werden soll - einerseits Ehre, die Reputationswährung des Heroischen, andererseits Mord, die Normverletzung des Verbrecherischen. Das daraus resultierende Oxymoron allerdings folgt einer ganzen Reihe analoger Formeln, alle gleichermaßen befremdlich, mit denen uns das Stück gleichwohl vertraut macht: „an honourable murderer“ ist kaum etwas anderes als das „civil monster“, von dem Iago zuvor spricht (IV 1, 60), oder auch als Lodovicos „noble Moor“ (IV 1, 256) beziehungsweise überhaupt the Moor of Venice, als der Othello ja gemeinhin gilt: eine Aufschichtung oder Verkettung von Oxymora, mit denen die Titelfigur 
durchweg belegt wird, um die Spannung, die sich in ihr verkörpert, rhetorisch zu markieren.

Die für das Thema dieses Bandes relevante Frage ist, ob die Formel ,Fremde Helden' ebenfalls ein solches Oxymoron bildet oder ob sie, wie im Weiteren argumentiert, nicht vielmehr ein Pleonasmus ist beziehungsweise eine analytische Aussage, die lediglich den beiden Begriffen offensichtlich Inhärentes darlegt. Diese Ansicht soll nun mit Blick auf den Stückanfang, dessen Handlungselemente Shakespeare nicht seinen Quellen entnommen, sondern weithin selbst erfunden hat, begründet werden. Wenn nämlich der Stückverlauf und insbesondere der mörderische Schluss den Vorgang einer Deheroisierung zeigt, dann ist der I. Akt nicht zuletzt deshalb so bemerkenswert, weil er eine reichlich unwahrscheinliche Heroisierung vorführt, und zwar explizit und ausschließlich durch Mittel des Erzählens. In diesem Zuge wird das Narrative, also das dem Drama eigentlich generisch Fremde, zugleich genutzt, so meine These, um Fremdheit als notwendige Bedingung für Heldentum zu etablieren.

3.

Was für Shakespeares Publikum ein Moor war, ist notorisch unbestimmt und unbestimmbar. ${ }^{18}$ Der Gebrauch des oft verwendeten Wortes schwankt zwischen geographischen, kulturellen, ethnischen und religiösen Kennzeichnungen, die teils ineinander übergehen, teils aber für uns ganz Unterschiedliches betreffen. Moor meint sowohl ,Nordafrikaner' wie ,Araber' als auch ,sub-saharische Afrikaner', meint insbesondere auch ,Muslim' oder generell ,Vertreter einer anderen Religion' wie überhaupt ,Orientale', ,Barbar oder ,alle außer uns'. Ganz ähnlich übrigens wie Turk ${ }^{19}$ dient Moor im frühneuzeitlichen Sprachgebrauch somit zur Bezeichnung generischer Fremdheit, für die Roderigo in der Auftaktszene die prägnante Formel bietet, wenn er von „an extravagant and wheeling stranger“ spricht „of here and everywhere" (I 1, 135-136). Othello ist damit der einzige Fremde im vielhundertköpfigen Shakespeare-Personal, der ausdrücklich so bezeichnet und dazu mit dem Akzent auf vagrancy versehen wird, also Vagabundieren, Wandern, rastlos Umherirren, nach zeitgenössischem Verständnis Merkmal des Unzivilisierten, Unzugehörigen und Ungehörigen, eben eines Moor.

Zugleich ist der Rekurs auf Orte eine zentrale Strategie zur Konstruktion von Fremdheit. Wie Bernhard Waldenfels in seiner Topographie des Fremden ausführt, ist dafür das Externum, das Anderswo, das Außerhalb zu dem Bereich, den wir zu un-

18 Siehe hierzu vor allem die Diskussionen in Michael Neill: Introduction. In: William Shakespeare: Othello, hg. von Michael Neill. Oxford 2006, 1-179, hier 113-130, sowie Vaughan: Othello (Anm. 1), 51-70.

19 Siehe Daniel Vitkus: Turning Turk. English Theatre and the Multicultural Mediterranea. Basingstoke 2003. Matthew Dimmock: New Turkes. Dramatizing Islam and the Ottomans in Early Modern England. Aldershot 2005. 
serem eigenen deklarieren, grundlegend: „Unter den Voraussetzungen begrenzter Ordnungen macht sich das Fremde bemerkbar in Form eines Außer-ordentlichen, das auf verschiedene Weise an den Rändern und in den Lücken der diversen Ordnungen auftaucht. ${ }^{\text {"20 }}$ Das Problem mit Othello, das in der Auftaktszene exponiert wird, liegt allerdings darin, dass dieser Außer-ordentliche, Extra-vagante offenkundig einen Ort im Zentrum der Stadtgesellschaft besetzt hält, buchstäblich das Bett der Senatorentochter, deren Körper zum Signum jenes reinen Gemeinwesens stilisiert wird, das nunmehr geschändet sei.

So jedenfalls die Reden von Iago und Roderigo, wenn sie Brabantio und damit uns zu Anfang von Othellos Tat erzählen und seine ,mohrische' Monstrosität mit drastischen Worten ausmalen. Drei Punkte sind an diesem Dramenanfang relevant: Erstens gilt Venedig, ein Schauplatz immerhin, der bereits im Stücktitel erwähnt wird, dem frühen 17. Jahrhundert ohnehin als kosmopolitische Stadt und damit Ansammlung von Fremden, in den Worten Thomas Coryates „a marketplace of the world“21, und als solche zugleich Spiegel sowie Wunschprojektion von Shakespeares London. Zweitens sind die beiden Wortführer der Fremdenfeindlichkeit, die offenkundig dennoch dort grassiert - übrigens auch gegenüber einem Florentiner wie Michael Cassio -, bezeichnenderweise selbst mit spanischen Namen belegt. Drittens ist alles fürchterlich Bedrohliche, ,Mohrische und Fremde an Othello, von dem hier erzählt wird, eben das: Erzählung, Zuschreibung, mutmaßlich Dämonisierung und Verleumdung, jedenfalls narrative Konstruktion, die, sobald er in der 2. Szene selbst auftritt, sofort widerlegt ist. Sobald nämlich die Titelfigur nicht mehr diegetisch vermittelt, sondern mimetisch präsentiert wird, erscheint sie als komplettes Gegenbild zum Ruf, der ihr vorauseilt (darin folgt das Drama Marlowes Tamburlaine-Tragödie ${ }^{22}$ ): souverän, beherrscht, selbstsicher, kultiviert und vor allem rhetorisch hoch versiert. Mit ihrer Eloquenz allein erwirbt sie sich Sozialprestige wie auch den Heldenstatus eines „noble Moor“, der zum Ende der Tragödie, wie zitiert, zerstört ist.

Ihre berühmteste Erzählszene bedarf daher erneuter Kommentierung: „Rude am I in my speech" (I 3, 82) - so beginnt Othello seine Rede vor dem Senat, wo Brabantio ihn der Hexerei und Zauberkunst, durch die er ihm die Tochter geraubt habe, anklagt: Othello bietet also eine klassische captatio in performativem Widerspruch zu seinem Verbalverhalten auf, denn mit der Behauptung seiner soldatischen Rauheit („little blessed with the soft phrase of peace“ [I 1, 83]) unternimmt der Redner nur ein viel erprobtes rhetorisches Manöver, um seine Lebensgeschichte als „round, unvarnished tale“ (I 1, 91) zu beglaubigen. Die vorgebliche

20 Bernhard Waldenfels: Studien zur Phänomenologie des Fremden, Bd. 1: Topographie des Fremden. Frankfurt am Main 1997, 10-11.

21 Thomas Coryate: [Description of Venice] from Coryate's Crudities, London 1611. In: William Shakespeare: The Merchant of Venice, hg. von Leah S. Marcus. New York 2006, 114-121, hier 116.

22 Siehe hierzu den Beitrag von Ralf Hertel in diesem Band. 
Schmucklosigkeit schmückt nämlich ungemein, und was als Selbstverteidigung beginnt, führt bald zu Selbsterhöhung und Heroisierung:

Her father loved me, oft invited me

Still questioned me the story of my life

From year to year: the battles, sieges, fortunes

That I have passed.

I ran it through, even from my boyish days

To th' very moment that he bade me tell it -

Wherein I spoke of most disastrous chances:

Of moving accidents by flood and field,

Of hair-breadth scapes i'th' imminent deadly breach,

Of being taken by the insolent foe

And sold to slavery; of my redemption thence,

And portance in my travailous history,

Wherein of antres vast and deserts idle,

Rough quarries, rocks, and hills whose heads touch heaven,

It was my hint to speak - such was my process -

And of the Cannibals that each other eat,

The Anthropophagi, and men whose heads

Do grow beneath their shoulders. This to hear

Would Desdemona seriously incline (I 3, 128-146)

Die so entfaltete „travailous history“ ist in zweifacher Hinsicht relevant: inhaltlich und performativ. Inhaltlich erweisen sich sämtliche Abenteuer und ethnographische Mitteilungen, wie oft bemerkt wurde, als Übernahmen aus der zeitgenössisch bekannten Literatur zur Natur- und Völkerkunde sowie aus Reiseberichten, zum Teil wörtlich anzitiert, wie beispielsweise Leo Africanus' Geographical Historie of Africa (englische Übersetzung von John Pory 1600), Plinius' Natural History (Philemon Holland 1601) und The Travels of Sir John Mandeville, jener populären Reisefantasie aus dem 14. Jahrhundert, in der auch die Acephali, die Plinius in Äthopien lokalisierte, ihren Auftritt haben. ${ }^{23}$ Kennzeichnend für den Zitatcharakter solcher Versatzstücke des Fremden ist hier zumal die terminologische Verschiebung von „Cannibals“ zu „Anthropophagi“, beides Hinweise auf Menschenfresser, die allerdings ganz unterschiedlichen Diskursformationen zugehören: ${ }^{24}$ canibales ist der bekannte Eigenname, den Kolumbus 1492 den Einwohnern des von ihm entdeckten Landes gab, die, wie er meinte, sämtlich Untertanen des Großen Khan seien und die, wie man ihm erzählte, ihre Feinde verzehrten; der Eigenname wurde daher generalisierend auf deren angebliche Ernährungspraxis übertragen. Umgekehrt verhält es sich bei den Anthropophagen, dem antiken Terminus für diese Praxis, der sich zum Beispiel bei Herodot findet und der sekundär auf bestimmte Völker, vornehmlich jenseits des Schwarzen Meeres angesiedelt, zur Kennzeichnung übertragen wurde.

23 Diese Angaben folgen Neill: Introduction (Anm. 18), 18-21.

24 Siehe die Diskussion dazu in Peter Hulme: Colonial Encounters. Europe and the Native Caribbean, 1492-1797. London 1986, insbesondere 70. 
Die Differenz von „Cannibals“ zu „Anthropophagi“ entspricht damit dem Unterschied von neuzeitlicher Geographie zu antiker Kosmographie, und dass Othellos Bericht diese Differenz einebnet oder übergeht, spricht daher ganz dafür, dass die Figur im Zeichensystem eben jener alten, überkommenen, moralisch grundierten und symbolisch konstruierten Weltordnung funktioniert, die mit den Entdeckungsreisen der Neuzeit fremd geworden ist. Wie John Gillies in diesem Zusammenhang bemerkt: „as in the ancient poetic geography, all Shakespearean moors combine a generic exoticism or exteriority with an inherent transgressiveness". ${ }^{25}$ Daher ist auch die Rhetorik von blackness, die Othello umgibt, nicht ethnisch oder ethnographisch zuzurechnen, sondern moralisch: black ist hier kein Antonym zu white, sondern zu fair, ${ }^{26}$ und die Insignia der Herkunft, mit denen er versehen ist, sind gleichfalls Merkzeichen und Manifestationen von Alterität. Auch das notorische Taschentuch ist kein einfacher Gebrauchsgegenstand, sondern Memento einer anderen Symbolordnung, von der nur noch erzählt werden kann.

Trotz aller Erzählungen aber, die Othello immer wieder aufbietet, trotz aller Hinweise auf die Konkreta seines Hintergrunds, ist entscheidend, dass alle genannten Einzelelemente kein kohärentes Bild ergeben, kein Itinerarium der von ihm zurückgelegten Reise und erst recht kein schlüssiges Indizienpuzzle zur Identifikation ihres Ausgangspunktes, seines Herkunftsorts. Anders als Eldred Durosimi Jones im Titel der genannten Pionierstudie nahelegt und anders als zum Beispiel Oroonoko, Aphra Behns Figur des „Royal Slave“ aus dem späten 17. Jahrhundert, ${ }^{27}$ hat dieser Moor kein kenntliches Zuhause. Othellos country bleibt Collage oder, anders gesagt, Kolportage aus diskursiven Versatzstücken, die wie Strandgut an die Küsten der Moderne schwemmen. Wenn er am Ende sein gesamtes Leben zur Seereise erklärt („Here is my journey’s end, here is my butt | And very sea-mark of my utmost sail“ [V 2, 266-267]), formuliert er das Prekäre einer Transitexistenz ohne festen Anker- oder Anlaufhafen. „Der Ort des Fremden in der Erfahrung“, schreibt Waldenfels, ,ist streng genommen ein Nicht-Ort. Das Fremde ist nicht

25 John Gillies: Shakespeare and the Geography of Difference (Cambridge Studies in Renaissance Literature and Culture; 4). Cambridge 1994, 25.

26 Siehe Leslie A. Fiedler: The Stranger in Shakespeare. London 1973, 171.

27 Siehe Aphra Behn: Oroonoko: or, the Royal Slave (London ca. 1678/88), hg. von Janet Todd. Harmondsworth [u.a.] 2003. Dazu folgender Kommentar von Gillies: Shakespeare and the Geography of Difference (Anm. 25), 28: „The difference between Othello and Oroonoko bespeaks a major paradigm-shift in the discursive construction of otherness between the beginning and end of the seventeenth century. Shakespeare's Renaissance imagination of otherness is still heavily indebted to the ancient poetic geography. Behn's Restoration idea of the other, however, is essentially modern and can readily be grasped in terms of postRenaissance forms of the discourses of race, slavery, the ,noble savage $[\ldots]$. The Elizabethan sense of the exotic (the ,barbarous, outlandish and strange ${ }^{6}$ ) might be characterized as ,hard', in the ancient sense of what Ben Jonson referred to as ,Magick, Witchcraft, or other such exotick arts'. By contrast, Behn's idea of the exotic might be characterised as ,soft', suggesting rather the fashionably strange or merely piquant." 
einfach anderswo, es ist das Anderswo". ${ }^{28}$ In diesem Anderswo ist auch the Moor of Venice heimisch.

Zugleich bezieht er daraus sein Prestige, und zwar allein durch narrative Übermittlung. Hier kommt die performative Hinsicht seines Senatsauftritts ins Spiel. Seine Rede dort geht nämlich weiter, indem der Redner die Wirkung dieser Art Erzählungen auf Desdemona beschreibt:

[...] My story being done,

She gave me for my pains a world of sighs:

She swore, in faith 'twas strange, 'twas passing strange,

'Twas pitiful, 'twas wondrous pitiful!'

She wished she had not heard it, yet she wished

That heaven had made her such a man; she thanked me,

And bade me, if I had a friend that loved her,

I should but teach him how to tell my story

And that would woo her. Upon this hint I spake.

She loved me for the dangers I had passed,

And I loved her that she did pity them.

This only is the witchcraft I have used. (I 3, 158-169)

Dabei überlagern sich die Wirkungsdimensionen. Was Othello von der appellativen Kraft seiner Abenteuererzählung berichtet, beglaubigt sich sowohl im Senatssaal („I think this tale would win my daughter too“ [I 3, 171], erklärt der Duke sogleich) als auch im Theater, denn als Zuhörer von Othellos Zuhörern ergeht es uns selbst kaum anders: Der Duke und Desdemona präfigurieren unsere eigene Reaktion. So setzt sich, was Brabantios Anklage als Hexerei bezeichnet, in die rhetorische Wirkmacht eines Redners um, der eben damit seinen Ausnahmestatus bestätigt, andererseits zugleich eine herausgehobene Stellung einnimmt - im Sinne charismatischer Ausstrahlungskraft -, und zwar ausscbließlich durch Narration. Und so holt das Drama alles Fremde, von dem erzählt wird, diegetisch ein. Es zeigt uns nichts, rein gar nichts von all den Abenteuern, exotischen Begegnungen und kriegerischen Taten, die Othello auszeichnen, ebenso wenig wie es seine Besuche im Hause Brabantios zeigt, die ja zweifellos besonders bühnenwirksam in Szene zu setzen gewesen wären. Es zeigt lediglich, dass er erzählt, wie er davon erzählt hat, und auf diese Weise seine Heldenposition gewinnt. Denn der venezianische Senat weist ja nicht nur die Anklage Brabantios ab, sondern überträgt dem einheimischen Fremden sogleich auch noch den Oberbefehl über die Armee, um das Gemeinwesen vor der Bedrohung durch externe Fremde zu verteidigen. Wenn das Heroische sich „essentiell performativ“ konstituiert, wie es in einem Freiburger Heldengrundsatzpapier heißt, dann in diesem Fall weniger durch „Ausführung von Heldentaten" als durch deren „Aufführung für andere“. ${ }^{29}$ Shakespeares Othello ist in erster Linie ein Erzählheld. 
Eben damit wird seine rhetorische Performance auch zur aemulatio eines der epischen Gründungshelden der Alten Welt, dessen Bedeutung jedem Renaissanceschuljungen präsent war. Mit der Erzählung seiner gefahrvoll-abenteuerlichen Irrfahrt durch exotisches Terrain, die seine noble Zuhörerin derart mitreißt, dass sie ihm zur Antwort darauf ihre Liebe eingesteht, rekapituliert Othello den Erzählakt, mit dem Aeneas einst die Liebe Didos auslöste und rekapituliert zugleich - und wichtiger - das rhetorische Verfahren, sich in einer akuten Verlegenheit auf klassische Präzedenzien und epische Helden zu besinnen. In den Worten von Lynn Enterline: „in his culturally and institutionally saturated citation of Roman eloquence the erring barbarian' performs what it means to be European" ${ }^{30}$ Mit seiner Vergil-Performance also qualifiziert der ,Mohr ${ }^{6}$ sich im Senat nicht nur zur Anerkennung durch den Staat, sondern zugleich für sämtliche Erhöhungsgesten bis hin zur Übertragung der Befehlsgewalt. Sein Heldentum gewinnt er ganz aus narrativer Zitation.

Wie bei aller Wiederholung allerdings bleibt dabei eine Differenz bestehen, ein konstitutiver Unterschied im Wiederholen, ein Residuum an Andersheit, das sich in der Performance gerade nicht umsetzt und die Repetition als solche markiert. Diese Differenz nun ist es, was Iago gleich zu Anfang ausweist und durchweg präsent hält und was das Stück mit dem Terminus blackness belegt. Es ist zugleich, so meine ich, die Bedingung für Othellos Heldentum, worin er sich von jenen „wealthy curlèd darlings of our nation“, von denen Brabantio schwärmt (I 2, 68), grundsätzlich unterscheidet - wie für das Heroische überhaupt, das ja, so die eingangs angekündigte Vermutung, durch Fremdheit notwendig bedingt ist. Denn in dem Maße, wie Othello im Weiteren von Iago verführt und verwirrt wird, verliert er schnell alles Heroische, wenn er sich zunehmend ins hässliche häusliche Eifersuchtsdrama verstrickt. Das ist es, was das Stück uns zeigt - im Unterschied zum Heldendrama, von dem es lediglich erzäblt -, ein Vorgang quälender Deheroisierung, in dessen Zuge der Protagonist nicht nur alle Eloquenz, ja seine Sprachfähigkeit einbüßt, sondern auch alle Markierungen des Außerordentlichen, Fremden: als geifernder, gewalttätiger Ehemann, der seine Frau misshandelt, ist er ins biederschlimme Alltagsmaß gerückt. „Größe ist, was wir nicht sind“, befand einst Jakob Burkhardt in seinen Weltgeschichtlichen Betrachtungen. ${ }^{31}$ Othello wäre demnach nur so lange groß, wie diese Differenz besteht, und eine Figur nur Held, solange sie uns fremd bleibt.

Ganz zum Ende der Tragödie, in Othellos letzten Worten, findet sich Bestätigung für diese Lesart. Denn hier findet der Held wieder zu seinem anfangs ausgewiesenen rhetorischen Vermögen, das heißt zum Modus des heroischen Erzählens

30 Lynn Enterline: Eloquent Barbarians. Othello and the Critical Potential of Passionate Character. In: Lena Cowen Orlin (Hg.): Othello: The State of Play. New York 2014, 149-176, hier 160.

31 Zitiert nach Pfister: Einführung (Anm. 11), 14. 
zurück, wenn er, wie erwähnt, seinen Nachruf regelt und dazu der Nachwelt eine weitere Episode seiner extra-vaganten Existenz berichtet:

I have done the state some service, and they know't -

No more of that. I pray you in your letters,

When you shall these unlucky deeds relate,

Speak of me as I am; nothing extenuate,

Nor set down aught in malice: then must you speak

Of one that loved not wisely, but too well;

Of one not easily jealous, but being wrought,

Perplexed in the extreme; of one whose hand,

Like the base Indian, threw a pearl away

Richer than all his tribe; of one whose subdued eyes,

Albeit unusèd to the melting mood,

Drops tears as fast as the Arabian trees

Their medicinable gum. Set you down this;

And say besides that in Aleppo once,

Where a malignant and a turbaned Turk

Beat a Venetian and traduced the state,

I took by th' throat the circumcisèd dog

And smote him - thus.

He stabs bimself. (V 2, 338-355)

Der Freitod zählt seit der Antike zu den gängigsten Maßnahmen zur - jedenfalls versuchten - Selbstheroisierung, denn anders als durch eigene Hand kann ein wahrer Held, dessen Stärke alle Gegenspieler übertreffen muss, nicht sterben. ${ }^{32}$ Das wahrhaft Bemerkenswerte aber an der hier erzählten Heldentat ist nicht nur, dass ihre Narration tatsächlich Performanz im Sinne echter Ausführung bedeutet - mit dem deiktischen „thus“ vollzieht der Sprecher ja, was er erzählt, und aktualisiert somit die berichtete Historie in einem ebenso gewaltigen wie gewalttätigen präsentischen Sprechakt -, sondern dass der Redner sich in einem abgründigen reenactment zugleich verfremdet und erneut heroisiert: Sein Dienst am Staat ist doppelt, denn mit der Erledigung des „turbaned Turk“ erledigt sich Othello jetzt gleich selbst. Der ,Mohr' von Venedig macht sich zu jenem ,ottomanischen' Feind von Venedig, auf den sein Name immer schon verweist, ${ }^{33}$ und eben dadurch zu Venedigs Retter, dass er beide, ,Mohr` und Türke, zugleich liquidiert. „Nicht das Blut [...] macht den Krieger zum Helden“, schreibt Münkler, „sondern seine Bereit-

32 Die andere traditionell verbürgte Todesart wahrer Helden ist durch Banalitäten (wie Tamburlaines Fieberanfall) oder feige Memmen, die eine kleine Schwachstelle des Heldenkörpers (wie Achilles' Ferse oder Siegfrieds Schulter) hinterrücks ausnutzen. Für diese Hinweise danke ich den Diskussionsteilnehmern der Freiburger Tagung im März 2015.

33 Siehe Michael Neill: Editorial Procedures. In: William Shakespeare: Othello, hg. von Michael Neill. Oxford 2006, 181-194, hier 194: ,the resemblance of ,Othello' to ,Othman' - the founder of the Ottoman (or ,Othoman') Turkish empire - is unlikely to be accidental and it lends additional irony to Othello's final self-identification with the ,malignant and ... turbaned Turk' (5.2.351) whose killing is reenacted in his own suicide“. 
schaft zum Selbstopfer, durch das andere gerettet werden" ${ }^{34}$ Diese stellt sich hier unter Beweis und restituiert den Heldenstatus. Der Held geht in die Geschichte ein, indem er förmlich in ihr aufgeht.

Zugleich reiteriert der Held hier seine Fremdheit, und zwar mit einer regelrechten Kette einschlägiger Kennzeichnungen, die er Lodovico als Nachrufregler in die Feder diktiert: Nicht genug mit „turbaned Turk“ und „circumcisèd dog“, Othello erklärt sich auch noch zum „base Indian“ (so in der Quarto-Ausgabe von 1622) oder aber, wie die Lesart der Folio-Ausgabe von 1623 lautet, „base Judean“ - eine der meistdiskutierten textuellen Varianten des gesamten Stücks. Doch diese Instabilität des Textes stärkt nur die Alterität dieses Helden, der sich in solcher Überdetermination - zugleich Türke, Inder, Indianer, Jude sowie Hund - als kategorial fremd darstellt. Damit zu einer knappen Schlussbetrachtung, die das mediale Kennzeichen von Bübnenhelden und noch einmal die Rezeptionsgeschichte von Othello in den Blick nimmt.

4.

Über kein Stück aus dem Shakespeare-Kanon sind aus dem 17. Jahrhundert mehr Aufführungsbelege überliefert, und keines hat seit jeher seine Zuschauer zu emphatischeren Reaktionen immer wieder neu herausgefordert, bis hin zu aktiven Eingriffen in die Bühnenhandlung. Davon berichtet bereits Samuel Pepys, der 1660 im Theater eine „very pretty Lady“ erlebt, „who [...] ,called out" in distress ,to see Desdemona smothered““. 35 Spektakulärer noch ist der Bericht Stendhals, der 1822 in Baltimore erlebt, wie ein amerikanischer Soldat aus dem Publikum auf den Darsteller des Othello schießt, weil er nicht tatenlos mit ansehen konnte, wie ein Schwarzer einer Weißen zusetzt. ${ }^{36}$ Solche leidenschaftlichen Publikumsreaktionen bis hin zur Grenzverletzung zwischen Spiel und Wirklichkeit spiegeln nur die ungeheuren Leidenschaften, die auf der Tragödienbühne vorgeführt werden und dieses Stück, was immer sonst es sein mag, zu einer grandiosen Affektstudie machen.

Nach der in diesem Beitrag unternommenen Lesart aber scheint es zweifelhaft, um nicht zu sagen: völlig verfehlt, diese Tragödie zur Affektstudie eines Afrikaners zu stilisieren, wenngleich ihre Verwicklung in rassistische Diskurse seit dem 19. Jahrhundert wohl unvermeidlich geworden ist. Gerade die emphatischsten unter den bekannten Zuschauerreaktionen (von denen die zitierten ja nur wenige Beispiele sind) aber lassen einen solchen Schluss nicht zu, und die Verlegenheit, in die der Titelheld, wie eingangs bemerkt, schwarze Zuschauer und Leser bringt, weist in eine andere Richtung: Solange sie in der Figur eine Darstellung als countryman se-

34 Münkler: Heroische und postheroische Gesellschaften (Anm. 7), 742.

35 Zitiert nach Lena Cowen Orlin: Introduction. In: Lena Cowen Orlin (Hg.): Othello: The State of Play. New York 2014, 1-16, hier 1.

36 Ebd. 
hen wollen oder müssen, kann diese ihnen gerade nicht modell- oder heldenhaft erscheinen, sondern präfiguriert lediglich die Pein von Assimilation. Dieselbe Verlegenheit trifft übrigens auch die großen schwarzen Schauspieler wie Ira Aldridge oder Paul Robeson, die mit den Triumphen, die sie in der Rolle Othellos feiern, immer auch Gefahr laufen, Shakespeares diskursive Konstruktion des ,Bühnenmohren' unfreiwillig zu authentifizieren, das heißt, durch ihre schiere Präsenz zu beglaubigen, was uns das Stück doch reflexiv vorführt: schwarze Körperlichkeit. Deshalb mag Othellos Bühnendarbietung in blackface, wie im gleichermaßen packenden wie peinlichen Auftritt von Laurence Olivier am National Theatre im Jahr vor Erscheinen von Jones' Studie, ${ }^{37}$ letztlich wohl die angemessenere, weil analytisch präzisere Theaterpraxis sein. Denn nicht als Charakterstudie eines ,Mohren“ oder Schaustück eines menschlich-allzumenschlich passionierten Afrikaners sollten wir Othello auffassen, sondern als Anatomie des Helden: im Sinne von Zergliederung einer Diskursfigur sowie Zurschaustellung ihrer Elemente.

Anders also als der Titel von Jones' Pionierbuch nahelegt, hat Othello keine countrymen. Er ist und bleibt „an extravagant and wheeling stranger“, Widerpart einer Mehrheitsgesellschaft, die ihn als Fremden aufnimmt und genau so lange als Helden feiern kann, wie seine Fremdheit narrativ beglaubigt wird. Anders aber auch als meine Titelformulierung nahelegt, ist diese Fremdheit nicht als skalierbare Größe zu nehmen, die mal mehr, mal weniger in Rechnung steht, sondern im Waldenfelsschen Sinne radikal und kategorial ist.

Deshalb verstehe ich den Ausdruck ,Fremde Helden auf der Bühne', der den Titel dieses Bandes abgibt, als eine analytische Aussage, und deshalb verstehe ich den Prozess von Othellos Heldenwerdung, wie wir ihn auf der Bühne mitverfolgen, als performativen Beleg dieses Urteils. Gerade die Aeneas-aemulatio führt ja auch vor Augen, wie eine solche Übernahme zugleich fehlschlägt und fehlschlagen muss, weil das Heroische zwar vorgängig, aber nicht einfach vorrätig ist und wie eine Maske aufzusetzen wäre, sondern unverfügbar, unvereinbar oder unberührbar bleibt: extra-vagant, außer-ordentlich, fremd.

Daher ist Othellos angestammter Ort kein anderer als die Bühne, denn das Theater ist ja selbst ein solcher Nicht-Ort, „of here and everywhere“, ein Ort des Fremden, der uns aus der Alltagsordnung ausnimmt, geradezu herausfallen lässt und wer weiß wohin versetzt, ja gewaltig mitreißt - oder wo sind wir eigentlich, wenn wir zusehen, wie eine unschuldige Frau von ihrem blindwütigen Gatten erwürgt wird?

37 Regie führte Stuart Burge; die Produktion wurde 1965 durch BHE Films verfilmt und ist so der Nachwelt bis heute zugänglich. 
